$(\mathrm{n}=2)$ and 30 minutes of NOS inhibition [with $1 \mathrm{mM}$ : L-NOrnithine, 1400W and S-Methyl-L-thiocitrulline] reduced baseline CBF by $20 \%$ to $9.6 \mathrm{~Hz} \mathrm{SD} \pm 0.9(\mathrm{p}<0.001)$ but the $\mathrm{P}_{2} \mathrm{X}_{4}$ inhibitor $[10$ $\mu \mathrm{M}$ brilliant blue $\mathrm{G}]$ had no effect.

Conclusion $\mathrm{P}_{2} \mathrm{X}_{4}$ and $\mathrm{nNOS}$ are expressed in human airway cilia but do not co-localise. NOS inhibition reduced CBF whilst $\mathrm{P}_{2} \mathrm{X}_{4}$ inhibition did not, suggesting that blocking $\mathrm{P}_{2} \mathrm{X}_{4}$ activity alone is not sufficient to modify NOS activity or CBF.

\section{P257 HUMAN CYTOMEGALOVIRUS BINDING TO NEUTROPHILS TRIGGERS A PRO-SURVIVAL SECRETOME THAT MODULATES MONOCYTE MIGRATION, ACTIVATION AND PHENOTYPE}

doi:10.1136/thoraxjnl-2012-202678.349

M Reaves, D Storisteanu, M Wills, ER Chilvers, AS Cowburn. University of Cambridge, Cambridge, UK

Neutrophils provide the body's first line of defence against invading pathogens. They respond to infection by releasing an array of chemokines, cytokines, and superoxide anions that initiate cascades of other immune mediators and cell types. Although the rapid response and flexibility of neutrophils make them an integral part of the body's immune system, human cytomegalovirus (HCMV), paradoxically, may use neutrophil activation for its own evolutionary advantage. Here we report that human peripheral blood neutrophils exposed to a clinical strain of HCMV display a profound survival phenotype that occurs independent of viral gene expression. The initial HCMV driven survival response was partially dependent on ERK1/2 activation and profoundly inhibited by inhibition of NF-kB. Intriguingly, this initial survival event triggered by virus binding was augmented by a cytokine mediated effect whereby supernatants from infected neutrophils provided uninfected neutrophils with substantial protection against apoptosis - a protection which was PI3K as well as ERK1/2 and NF-kB dependent.

Concomitant with a transferable survival effect the HCMVneutrophil secretome also markedly manipulated autologous donor monocytes. Enhanced migration and subsequent differentiation to a permissive phenotype for HCMV infection was suggestive of a mechanism for efficient viral dissemination from the site of initial infection. Fascinatingly, although differentiation to a permissive phenotype was observed this was concomitant with downregulation of a number of key activators of the adaptive immune response. Overall, these data illustrate the manipulation of an anti-viral response by a pathogen to enhance the outcome of infection which, intriguingly, involves a cell type not productively infected by the pathogen itself. These data further illustrate the complexity of pathogen interactions with the host immune system as well as providing new clues into the mechanisms HCMV exploits for efficient viral dissemination which could have implications on our understanding for HCMV pathogenesis.

\section{Improving the care of sleep apnoea}

\section{P258 CHANGE IN SLEEP STUDY AND CPAP PROVISION FOLLOWING THE NIHCE CPAP TA}

doi:10.1136/thoraxjnl-2012-202678.350

${ }^{1} \mathrm{G}$ Hill, ${ }^{2} J R$ Stradling. ' On behalf of the Sleep Apnoea Trust Association, PO Box 60 , Chinnor, OX39 4XE, UK; ${ }^{2}$ Xxford Centre for Respiratory Medicine, Churchill Hospital, OXford OX3 $7 L J, U K$

Introduction We have assessed whether Primary Care Trusts' (PCTs) commissioning of sleep services has improved following the NIHCE 2008 technology appraisal on CPAP.
Methods We wrote to all PCTs in England, (under the Freedom of Information Act, 2000) for information on sleep studies commissioned, and CPAP prescriptions issued, for the years ending 31 March, 2008, 09, and 10. The PCTs were also asked who provided sleep studies or CPAP on their behalf (NHS hospital, GP, private, or other provider). Some PCTs did not reply, or claimed not to hold data, so we contacted appropriate NHS hospitals to obtain further information. Sleep study data was only obtained from approximately $75 \%$ of PCTs or associated hospitals. An alternative set of data, on the Department of Health (DH) website, was also used. Limited sales data from CPAP companies was also available for comparison.

Results In almost all PCTs, sleep studies and CPAP provision were from NHS hospitals. The incomplete data from PCTs showed that sleep studies rose from about 30,000 in 2007/8 to 48,000 in 2009/10, a 3 -year increase of about $60 \%$. Data on sleep studies published by DH rose from over 61,000 in $2007 / 8$ to over 86,000 in $2009 / 10$, a 3 -year increase of $41 \%$. For CPAP prescriptions issued, only $66 \%$ of PCTs submitted data for $2007 / 8$, rising to a $75 \%$ response by $2009 / 10$. On the basis of this incomplete information and with some assumptions, annual CPAP prescriptions rose from less than 17,000 in $2007 / 8$ to over 37,800 by $2009 / 10$, a three year increase of $126 \%$. There may be some under-reporting in the earlier years, and the industry sources suggested a 3 -year lower increase of nearer $80 \%$. In addition there are likely to be errors of coding. However, there was wide variation between PCTs suggesting patchy performance.

Conclusions We believe that the results show a clear improvement in the number of sleep studies and CPAP prescriptions over this period. Thus, following the NIHCE TA there has been an improvement in patient access to the diagnosis and treatment of sleep apnoea, though we are concerned that the wide variation suggests there is a substantial element of post-code lottery.

\section{P259 PREFERENCE OF PATIENTS WITH OBSTRUCTIVE SLEEP APNOEA SYNDROME (OSAS) FOR CONVENTIONAL CONTINUOUS POSITIVE AIRWAY PRESSURE(CPAP) OR BI-LEVEL CPAP (CFLEX) AND CHANGES IN RESISTANCE TO EXPIRATION}

doi:10.1136/thoraxjnl-2012-202678.351

'S Thomas, ${ }^{2} \mathrm{~A}$ Daya, ${ }^{1} \mathrm{HR}$ Gribbin. 'Sleep Clinic, James Cook University Hospital, Middlesbrough TS4 3BW, UK; ${ }^{2}$ Medical Physics Department, James Cook University Hospital, Middlesbrough TS4 3BW, UK

CFlex (Respironics) Bi-Level device lowers expiratory PAP (EPAP) early in expiration returning EPAP to the set inspiratory PAP (IPAP) before the start of the next inspiration. Most patients with OSAS will express a preference for either CPAP or CFlex at the time of treatment initiation. We investigated the possibility that choice of CFlex was related to interaction of breathing pattern and the CFlex device in 43 newly diagnosed patients with OSAS in a single-blind study. Inspiratory and expiratory time ( $\mathrm{Ti}, \mathrm{Te}$ ) were measured by rib-cage and abdominal inductance belts. IPAP and EPAP were measured at the mask by pressure transducer. In 23 patients we recorded simultaneous flow in the CPAP circuit and by using mask specific leak rates derived values for inspiratory tidal volume (Vt insp): ( $\mathrm{Vt}$ insp $=($ Mean Inspiratory Circuit Flow $x$ Ti) $-($ Mask leak $x$ Ti) Patients tried CPAP or CFlex in random order for periods of 10 minutes or until breathing was stable. One minute epochs of stable breathing were used to calculate mean values for Ti, Te, IPAP, EPAP and Vt insp

Results 19 patients stated a preference for CFlex (CFlexpref), 20 for CPAP (CPAPpref). 4 had no preference and received CPAP. We included them in the CPAP. pref group for analysis. For the whole group $(n=43)$ there was a small but significant fall in mean EPAP on CFlex compared with mean EPAP on CPAP (10.4 vs $11.5 \mathrm{~cm} \mathrm{H} \mathrm{H}_{2} \mathrm{O}$ ). 